\title{
Cartographic support for assessing the suitability of land for agricultural production in the PRC's Capital Region
}

\author{
Wang Haoyang, Elena Stanis*, and Polina Drygval \\ Peoples Friendship University of Russia (RUDN University), Faculty of Ecology, 6 Miklukho- \\ Maklaya St, Moscow, 117198, Russian Federation
}

\begin{abstract}
This article examines the role of meteorological factors in the formation of adverse conditions for agricultural development in the capital region of the People's Republic of China (PRC). This area is one of the most densely populated, and the problem of food supply is very acute. The suitability of the area for agricultural activities not only depends on hydrometeorological conditions, but also on the frequency of meteorological disasters. Such disasters include droughts, flooding of the territory due to intensive precipitation and extremely high air temperatures. In this work, it has been made an attempt to analyse the frequency of adverse events of meteorological phenomena and their distribution over the territory of the capital region. Electronic maps of frequency of both individual disasters and an integral map were created, which allowed dividing the territory into 4 categories of total adverse hydrometeorological factors for the development of agricultural production. This study can be as an additional basis for the planning and development of various sectors of agricultural production and for the implementation of measures to reduce the impact of meteorological disasters on agriculture.
\end{abstract}

\section{Introduction}

The «Capital Region» of China comprises three provincial administrative regions Beijing, Tianjin and 11 county-level cities located in Hebei Province. The Beijing-TianjinHebei region is located in North China, with a geographical range: $36^{\circ} 05^{\prime} \sim 42^{\circ} 37^{\prime}$ north latitude, $113^{\circ} 11^{\prime} \sim 119^{\circ} 45^{\prime}$ east longitude, an area is about $216,000 \mathrm{~km} 2$.

The climate type of all the study area is a temperate monsoon climate. All four seasons are pronounced in the central region of the PRC. This climate type is characterized by hot and rainy summers and fairly cold winters. Spring and autumn are comparatively short here [1].

The study area is very densely populated, and the problem of food supply is very acute. The intensification of agricultural production has reached a very high level in the PRC, and there is a dramatic shortage of land suitable for agriculture [2]. The suitability of the area

${ }^{*}$ Corresponding author: stanis-ev@,rudn.ru 
for such activities does not depend on hydrometeorological conditions and the frequency of meteorological disasters.

Due to many factors such as geographical location, topography, climate, geological structure [3], meteorological disasters such as high air temperature, floods, droughts often occur in the study area. These disasters affect not only on the lives of the residents but also the local agricultural production.

There have been no previous studies on a comprehensive assessment of the suitability of land in the PRC capital region for agricultural production, depending on climatic and meteorological factors, using mapping methods. This is the first time such an assessment has been carried out.

\section{Methods and materials}

\subsection{Interpolation method.}

The data for the study area come from 25 weather stations (observation points), spatial interpolation is required to describe the frequency of disasters in the whole area [4].

There are many spatial interpolation methods such as Inverse Distance Weighting (IDW), Kriging method, Tyson polygon method, etc. These methods are implemented using sophisticated mathematical and cartographic algorithms.

The Kriging method is used in this article. This method is widely used for spatial interpolation of different types of observations, such as ecological studies of air pollution and soil pollutants, observation of temperatures and precipitation when there is no regular spatial regular network of observations. This method takes into account spatial correlation and the result is more realistic and reliable. One mechanism that produces more reliable results is to remove trends and obtain weighted parameters, i.e. to account for the nonuniformity of the observation network thanks to a certain mathematical procedure.
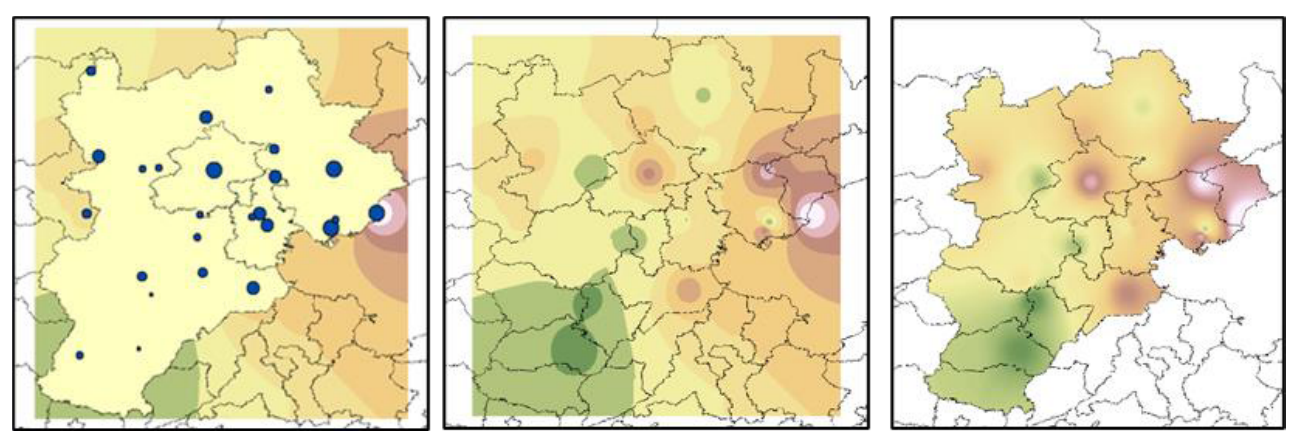

Fig. 1 Interpolation sequence in ArcMap 10.2.

Kriging is similar to interpolation IDW in that it weights the surrounding measured values to obtain parameter values where no measurements are available. The basic formula for these two interpolation tools is formed as a weighted sum of the data:

$$
\hat{Z}\left(\mathrm{~s}_{\mathrm{o}}\right)=\sum_{I=1}^{N} \lambda_{\mathrm{i}} \mathrm{Z}\left(\mathrm{s}_{\mathrm{i}}\right)
$$

where: $\mathrm{Z}(\mathrm{si})$ is the measured value at point $\mathrm{i}, \lambda \mathrm{i}$ is the weight of the measured value at point $\mathrm{i}, \mathrm{s} 0$ is the location of the predicted point , $\mathrm{N}$ is the number of measured values. The weighting coefficient, directly calculated by the computer in an appropriate program. [5-7]. 


\subsection{Criteria for assessing meteorological hazards}

\subsubsection{The frequency of drought occurrence}

Drought in the capital region mainly occurs in spring due to low rainfall. Although many drought-tolerant crops, such as cotton, are grown here, the occurrence of drought often affects crop quality.

Drought disasters are mainly caused by abnormal precipitation. Therefore, anomalous low precipitation is an important indicator for assessing drought risk. The calculation can be done using the formula proposed by the National Climate Centre of the PRC and published in the "Meteorological Drought Standards":

$$
\mathrm{PA}=\frac{P-\bar{P}}{\bar{P}} \times 100 \%, \bar{P}=\frac{1}{n} \sum_{i=1}^{n} P i
$$

where, PA - percentage of precipitation anomalies; P - precipitation amount $(\mathrm{mm}) ; \bar{P}_{-}$ average precipitation amount $(\mathrm{mm})$.

Table 1. PA drought type class

\begin{tabular}{|c|c|c|c|c|}
\hline \multirow{2}{*}{ Class } & \multirow{2}{*}{ Type of drought } & \multicolumn{3}{|c|}{ Percentage of anomalous rainfall \% } \\
\cline { 3 - 5 } & & Monthly scale & Seasonal scale & Annual scale \\
\hline 1 & Not available & $-40<\mathrm{PA}$ & $-25<\mathrm{PA}$ & $-15<\mathrm{PA}$ \\
\hline 2 & Weak & $-60<\mathrm{PA} \leq-40$ & $-50<\mathrm{PA} \leq-25$ & $-30<\mathrm{PA} \leq-15$ \\
\hline 3 & Average & $-80<\mathrm{PA} \leq-60$ & $-70<\mathrm{PA} \leq-50$ & $-40<\mathrm{PA} \leq-30$ \\
\hline 4 & Strong & $-95<\mathrm{PA} \leq-80$ & $-80<\mathrm{PA} \leq-70$ & $-45<\mathrm{PA} \leq-40$ \\
\hline
\end{tabular}

In this work, a monthly scale has been chosen. If the PA of a particular month is less than $-80 \%$, it means that the month is dry. If there are 3 or more dry months in a year, we call it a dry year.

\subsubsection{The frequency of flood occurrence}

Under the influence of monsoon climate precipitation in Beijing-Tianjin-Hebei region (capital region of PRC) is relatively concentrated. Precipitation is concentrated in BeijingTianjin-Hebei region - in July and August and accounts for $80 \%$ of the total annual precipitation.

If the precipitation exceeds $250 \mathrm{~mm}$ for 10 consecutive days in a year or $350 \mathrm{~mm}$ for 20 consecutive days in a given year, the year is classified as a flood year, according to the Ministry of Natural Resources of the PRC document "Technical Guidelines for Assessment of Suitability of Resource and Environmental Development and Land Development from Space".

\subsubsection{The frequency of high temperature occurrence}

A temperate monsoon climate is characterized by high summer temperatures. The highest temperature in the capital region occurs in summer and is about $40{ }^{\circ} \mathrm{C}$. The impact of high temperatures on agricultural production is also adverse.

If the temperature exceeds $35^{\circ} \mathrm{C}$ for 3 consecutive days or the temperature exceeds 35 ${ }^{\circ} \mathrm{C}$ for 2 consecutive days and on one of the days $>38^{\circ} \mathrm{C}$, we can say that the process is high temperature. If a high-temperature process occurs three times in a year, we will mark that year as high-temperature. The high temperature criterion is also given in the "Technical Guidelines for Assessing the Suitability of Resource and Environmental Development and Land Development from Space". 
The table below shows the number of dry years, the number of years with flooding, with high temperatures and the frequency of cases per year at 25 observation points from 1985 to 2018 (34 years). The frequency is calculated based on data from the region's weather bureau. (Database: Precipitation and temperatures for 1985-2018 in the PRC capital region).

Table 2. Frequency of adverse meteorological events occurrence

\begin{tabular}{|c|c|c|c|c|c|c|c|}
\hline \multirow{2}{*}{ № } & Station & \multicolumn{2}{|c|}{ Dry years } & \multicolumn{2}{c|}{ Years of flooding } & \multicolumn{2}{c|}{$\begin{array}{c}\text { Years with a high } \\
\text { temperatures }\end{array}$} \\
\cline { 2 - 8 } & $\begin{array}{c}\text { Observation } \\
\text { points }\end{array}$ & $\begin{array}{c}\text { Number } \\
\text { of events }\end{array}$ & Frequency & $\begin{array}{c}\text { Number } \\
\text { of events }\end{array}$ & Frequency & $\begin{array}{c}\text { Number } \\
\text { of events }\end{array}$ & Frequency \\
\hline 1 & Bazhou & 24 & 0.71 & 0 & 0.00 & 26 & 0.76 \\
\hline 2 & Baodi & 23 & 0.68 & 3 & 0.09 & 26 & 0.77 \\
\hline 3 & Baoding & 27 & 0.79 & 2 & 0.06 & 29 & 0.84 \\
\hline 4 & Beijing & 7 & 0.21 & 0 & 0.00 & 23 & 0.68 \\
\hline 5 & Potou & 28 & 0.82 & 3 & 0.09 & 28 & 0.81 \\
\hline 6 & Chengde & 15 & 0.44 & 2 & 0.06 & 13 & 0.38 \\
\hline 7 & Fengning & 9 & 0.26 & 0 & 0.00 & 10 & 0.28 \\
\hline 8 & Huailai & 16 & 0.47 & 1 & 0.03 & 14 & 0.4 \\
\hline 9 & Huanghua & 17 & 0.5 & 0 & 0.00 & 31 & 0.9 \\
\hline 10 & Leting & 18 & 0.53 & 7 & 0.2 & 24 & 0.71 \\
\hline 11 & Miyun & 14 & 0.41 & 0 & 0.00 & 13 & 0.39 \\
\hline 12 & Nangong & 14 & 0.41 & 0 & 0.00 & 28 & 0.81 \\
\hline 13 & Qinhuangdao & 8 & 0.24 & 10 & 0.29 & 17 & 0.5 \\
\hline 14 & Qinglong & 19 & 0.56 & 1 & 0.029 & 4 & 0.1 \\
\hline 15 & Raoyang & 10 & 0.29 & 2 & 0.06 & 27 & 0.79 \\
\hline 16 & Tangshang & 22 & 0.65 & 3 & 0.09 & 21 & 0.6 \\
\hline 17 & Tanggu & 22 & 0.65 & 0 & 0.00 & 22 & 0.63 \\
\hline 18 & Tianjin & 13 & 0.38 & 0 & 0.00 & 20 & 0.58 \\
\hline 19 & Weichang & 6 & 0.18 & 0 & 0.00 & 15 & 0.44 \\
\hline 20 & Weixian & 12 & 0.35 & 3 & 0.09 & 19 & 0.56 \\
\hline 21 & Xingtai & 16 & 0.47 & 5 & 0.15 & 31 & 0.92 \\
\hline 22 & Yanqing & 13 & 0.38 & 0 & 0.00 & 12 & 0.36 \\
\hline 23 & Zhangbei & 11 & 0.32 & 2 & 0.06 & 5 & 0.16 \\
\hline 24 & Zhangjiakou & 22 & 0.65 & 13 & 0.38 & 8 & 0.25 \\
\hline 25 & Zunhua & 7 & 0.21 & 7 & 0.21 & 10 & 0.3 \\
\hline & & & & & & & \\
\hline
\end{tabular}

\section{Results and discussion}

After filtering and analysis of the above data, all information was displayed in digital maps, using geoinformation system software (Arsgis 10.2).

The frequency of drought, the frequency of land flooding and the frequency of high temperatures in the PRC capital region were mapped (Figure 2, 3, 4). All factors were divided into 5 levels according to the severity of each meteorological factor for agriculture area: very low, low, medium, high, and very high. It is shown in Fig. 2, 3, 4. 


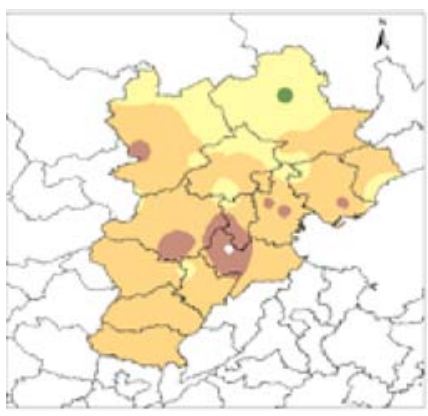

(a)

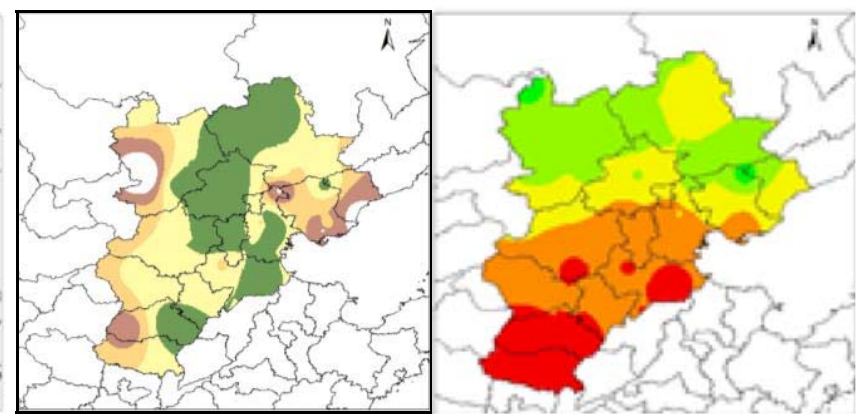

(b) (c)

Fig. 2: Maps of occurrence frequency: drought (a), floods (b) high temperatures (c) in capital region.

According to the frequency of the meteorological adverse events' occurrence, the results were ranked according to the assigned scores, which are shown in Table 3.

Table 3. The scoring table

\begin{tabular}{|c|c|}
\hline $\begin{array}{c}\text { Frequency of the meteorological } \\
\text { adverse events' occurrence }\end{array}$ & Score \\
\hline$<0.2$ & 6 \\
\hline $0.2-0.4$ & 4 \\
\hline $0.4-0.6$ & 2 \\
\hline $0.6-0.8$ & 1 \\
\hline$>0.8$ & 0 \\
\hline
\end{tabular}

The formula for assessing the suitability of land in the PRC capital region based on the frequency of a meteorological disaster occurrence:

$$
M B=B_{1}+B_{2}+B_{3}
$$

where MB-sum of suitability score; B1 - the frequency of drought occurrence; B2 - the frequency of flood occurrence; B3 - the frequency of high temperature occurrence. The formula for land suitability as a direct sum has been proposed by the authors. This is due to the fact that the authors think that all the parameters considered in this article play the same role in creating the degree of suitability of the territory of a capital region for agricultural production.

After overlaying the maps in Arcgis 10.2. using the «Raster Calculator» to add up the value of each grid cell of the three maps, a map of the land suitability of the PRC capital region for agriculture, taking into account the frequency of meteorological disasters, was obtained (Figure 3). 


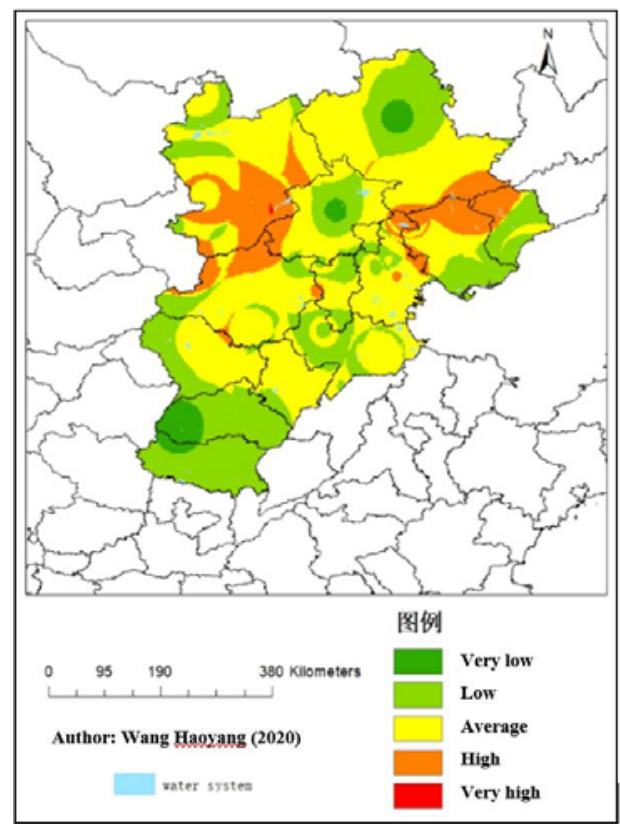

Fig. 3. Map of land suitability of the PRC capital region for agricultural production based on the frequency of meteorological disasters

Based on the results, the following points should be made: the overall frequency of droughts in the study region is medium (moderate). The frequency of droughts in the north is generally lower than in the south, and the droughts themselves are more frequent in spring, which is due to the anomalous low number due to the region's monsoon climate. Flooding is also associated with the monsoons, which occurring more frequently in the western and eastern parts of the region. But in general, the frequency of flooding in the central region is low. The frequency of anomalous high temperatures in the region gradually decreases from south to north, that consistent with climatic zonality.

\section{Conclusions}

The land suitability map of the PRC capital region based on the frequency of meteorological disasters and it derived from this stud. Map shows that the overall suitability of the area in the region is medium. At the same time, the integral suitability of conditions for agricultural production in the south is low due to high temperatures and frequent drought disasters. The greatest suitability of meteorological factors for agriculture is in the eastern and western parts of the study area, although the area is small [8].

Based on the results of the analysis and the study the following suggestions can be made for the region: the area of the capital region needs further justified land reclamation with the construction of a modern closed irrigation system and water saving reservoirs.

Further development of meteorological disaster monitoring and forecasting using remote sensing technology will make it possible to carry out the necessary measures to prevent agricultural emergencies in advance [9-10].

This paper has been supported by the RUDN University Strategic Academic Leadership Program. 


\section{References}

1. Han Jiahao, Zhang Qi, Shen Lingling. Journal of Yangtze River academy of Sciences, 1-7 (2019)

2. Jiang Li, Jiang Jing, Agricultural Economics and Management, 5,32-45 (2015)

3. MA Zhen, XIE Hailan, LIN Liangjun, HU Qiuyun, QIAN Yong, ZHANG Surong, WANG Guiling, LI Jianguo, TAN Chengxuan, GUO Haipeng, ZHANG Fucun, ZHAO Changrong, LIU Hongwei, Geology in China, 44(5), 857-873 (2017)

4. Zou Wen-yue, Yin Shui-qing,Wang Wen-ting, Journal of Hydrology, 598, (2021)

5. W. Zhang, K. L. Wang, H. S. Chen [and all] J. Sci. Food Agric., 92, 1094-1102 (2012)

6. A.P. Handayani, A. Deliar, I. Sumarto. I. Syabri, Indonesian J. of Geography, 52(1) (2020)

7. Yongxing Wang, Gang Hua, Weige Tao,Lei Zhang, Wireless Personal Communications, 115 (2020) (prepublish).

8. Qu Chongchong, Wang Jing, he Mingke, Operations research and management, 30 (01), 36-42 (2021)

9. Zhang Yaowen, Li Haijun, Li Hao, Zhang ruilei, Hydropower energy science, 38(10), 44-47 (2020)

10. Gao Junhai, Journal of natural disasters, 29 (04), 1-7 (2020) 